\title{
Requests for electroencephalography in a district general hospital: retrospective and prospective audit
}

\author{
D Smith, R Bartolo, R M Pickles, B M Tedman
}

Department of

Neurology, Walton

Centre for

Neurology and

Neurosurgery,

Fazakerley,

Liverpool L9 7LJ

D Smith

consultant neurologist

EEG Department,

Wrexham Maelor

Hospital, Wrexham

LL13 7TD

R Bartolo

EEG technician

R M Pickles

general practitioner

Department of

Neurophysiology,

Walton Centre for

Neurology and

Neurosurgery

B M Tedman

consultant

neurophysiologist

Correspondence to:

D Smith

brown-s@wcnn-tr.

nwest.nhs.uk

BMJ 2001;322:954-7

\begin{abstract}
Objectives To determine the number of inappropriate requests for electroencephalography (EEG) and whether guidelines on use could reduce this number.

Design Audit with retrospective and prospective components.

Setting EEG department in district general hospital and centre for neurology and neurosurgery.

Participants Retrospective: 368 at the general hospital and 143 patients at the neurology centre. Prospective: 241 patients undergoing EEG at the general hospital.

Interventions Guidelines for EEG issued to users of service at the general hospital.

Outcomes Retrospective: differences in requesting practice, result in different clinical scenarios, relative roles of procedure, clinical acumen in establishing diagnosis, usefulness of procedure. Prospective: change of requesting practice, impact on use.

Results There were considerable differences in requesting practice. Non-specialists seem to use EEG as a diagnostic tool, especially in patients with "funny turns," when it is much more likely to yield potentially misleading than clinically useful information. The overall proportion of procedures considered to influence management, to be justifiable, and to be inappropriate were $16 \%$ (59), 28.3\% (104), and 55.7\% (205), respectively. In the prospective study the total number of requests was significantly reduced $\left(\chi^{2}=33.85, \mathrm{df}=5, \mathrm{P}<0.0001\right)$, mainly because of fewer requests in patients with non-specific "funny turns" $\left(\chi^{2}=21.90, \mathrm{df}=6, \mathrm{P}=0.0013\right)$. There was a concomitant change in the usefulness of EEG $\left(\chi^{2}\right.$ 26.99, $\mathrm{df}=2, \mathrm{P}<0.0001)$.

Conclusions This original audit informed clinical practice and had potential benefits for patients, clinicians, and provision of service. Systematic replication of this project, possibly on a regional basis, could result in financial savings, which would allow development of accessible local neurophysiology services.
\end{abstract}

\section{Introduction}

Electroencephalography (EEG) has been readily available to clinicians throughout the United Kingdom for more than 30 years. It has gained a reputation as a diagnostic test with a range of indications, but its real uses and limitations are not widely appreciated and its usefulness in clinical practice has never been properly evaluated.

\section{What can EEG do?}

A single interictal EEG often helps to classify epilepsy ${ }^{23}$ and can provide support for a diagnosis of epilepsy when the clinical features are highly suggestive. However, EEG is rarely, if ever, the sole determinant of this diagnosis. ${ }^{4}$ EEG can help to predict the risk of recurrence after a first seizure ${ }^{5}$ and the risk of relapse after drug withdrawal. ${ }^{6}$ Uncommon indications include the differentiation between functional psychoses (presenting with altered consciousness) and organic confusional states, and demonstration of characteristic periodic discharges in rare encephalopathies (subacute sclerosing panencephalitis, sporadic or acquired Creutzfeldt-Jakob disease). A single procedure cannot diagnose or exclude epilepsy, assess the severity of epilepsy or the response to treatment, ${ }^{7}$ diagnose or exclude the presence of a brain tumour, or differentiate dementia from pseudodementia. ${ }^{4}$

A single procedure can also produce misleading information. ${ }^{4}$ While it can show specific epileptiform discharges in many patients with epilepsy, ${ }^{8}$ similar discharges are seen in $10 \%$ of patients who have undergone intracranial surgery and 3\% of individuals with psychiatric disorders who do not have epilepsy. ${ }^{9}$ Though these specific discharges are rarely seen in people without symptoms,${ }^{10}$ it is crucially important to recognise that normal phenomena, artefacts, and nonspecific abnormalities, occurring in about $20 \%$ of the general population, are open to misinterpretation and yield false positive results. ${ }^{11}$

Is EEG being used effectively?

There is unrestricted access to EEG in most medium sized district general hospitals throughout the United Kingdom. Most requests for EEG come from non-specialists, ${ }^{4}$ and only 60 out of 250 neurophysiology departments in the United Kingdom are staffed by adequately trained neurophysiologists (personal communication). Therefore there is considerable potential for unnecessary requests and misinterpretation of the results.

Not much has been published on the use of EEG in routine clinical practice. In 1995 Nicolaides et al reported an audit of requests for EEG in general paediatrics. ${ }^{12}$ Two fifths of requests were considered to be inappropriate, and 50-60\% of referring doctors thought that the procedure could diagnose or exclude epilepsy. The authors concluded that "despite guidelines clinicians remained uncertain about when and for whom to request an EEG." In 1990 Binnie observed that $60 \%$ of referrals to a specialist epilepsy centre were "routine EEG to assess control" in patients with epilepsy and found that results influenced management in only $3 \% .^{13}$ When referral policy was changed the proportion of routine procedures fell to $15 \%$ and $40 \%$ influenced management. The freed technical capacity was used to expand telemetry services, in which the success in answering the question that prompted referral rose from $67 \%$ to $91 \%$.

The limited evidence suggests that misconceptions about the diagnostic capability of EEG are common and, consequently, its use is suboptimal. However, changes in practice are possible. We carried out an audit of requests, from various sources, in a service 
which is typical of that available in district general hospitals throughout the United Kingdom.

\section{Methods}

\section{Retrospective audit}

We reviewed the case notes and request forms of 368 patients who underwent EEG in one district general hospital between August 1994 and January 1995. In the four patients who had more than one procedure we included only the first record.

We collected data on source of request (general practitioner, neurologist, physician, paediatrician, psychiatrist, other) and reason (epilepsy, seizures, funny turns (episodes of altered consciousness, awareness or behaviour, aggressive behaviour, rapidly progressive dementia, acute confusional states, mental symptoms, including gradual cognitive decline), and "other") for request.

We noted the expectation of the requesting clinician (for example, "blackouts-exclude epilepsy"). The result was recorded as normal, non-specifically abnormal, epileptiform (focal sharp spike or generalised spike and wave), or other specific finding-for example, encephalopathic. The working diagnosis, before and after procedure, was drawn directly from the notes.

\section{Analyses}

We analysed the data to establish if there were differences in requesting practice among users of the service. We also determined the quality of information provided by single interictal EEG in various scenarios. We assessed the role of EEG in establishing the diagnosis. This was categorised as classification of epilepsy, diagnosis of epilepsy, diagnosis of another specific condition, and as justifiable or inappropriate. We considered that requests were inappropriate when a diagnosis had been made on clinical grounds and the subsequent ordering of an EEG could not have produced useful information or when there had been an unsatisfactory attempt to achieve a clinical diagnosis, usually failure to obtain an eyewitness account of the patient's attacks. We assessed the usefulness of the procedure, defined as influencing management, (classification or diagnosis of epilepsy, diagnosis of another specific condition, and when a correctly interpreted EEG influenced decision making), justifiable, or inappropriate.

For the purposes of setting standards we applied the same process to the case notes and EEG forms of 143 patients who had EEG performed at a regional neurosciences centre during October and November 1995.

\section{Intervention}

We presented the results of the retrospective audit to the clinicians at two district general hospitals. At these meetings we presented guidelines and circulated them to all doctors using the service. Under the heading "indications" we included several clinical categories. Potential findings and their impact on management decisions were explained-for example, in someone with a confident diagnosis of epilepsy which was difficult to classify on clinical grounds an EEG revealing either a focal or generalised epileptiform abnormality would assist classification and choice of treatment. Similarly we categorised "not indications" and discussed misconceptions underlying common reasons for requests-for example, "blackouts, exclude epilepsy," where a normal reading does not exclude epilepsy and overinterpretation of non-specific abnormalities carries a risk of misdiagnosis.

\section{Prospective audit}

Referring clinicians had agreed to try to reduce the proportion of inappropriate requests to between 35\% and $40 \%$. The prospective audit entailed the review of notes and request forms from 241 patients undergoing EEG between March and August 1996. No patients had more than one EEG. No patients underwent EEG in both the retrospective and prospective components of the audit. We used $\chi^{2}$ tests to assess association between the group (retrospective, prospective) and categorical variables such as requesting clinician, reason for request, results, and usefulness.

\section{Results}

\section{Retrospective audit}

Reasons for requesting EEG varied between clinicians. While neurologists concentrated on patients with epilepsy and seizures (62\%), other doctors used the procedure as a diagnostic tool-for example, 134/166 $(81 \%)$ patients with "funny turns" or aggressive outbursts were referred for "exclusion of epilepsy." In these 166 patients the results were normal in 107 $(65 \%)$, non-specifically abnormal in $42(25 \%)$, and showed epileptiform discharges in only three $(2 \%)$. Three children who presented with inattentiveness had generalised spike and wave, indicating a diagnosis of childhood absence epilepsy.

The procedure influenced management in 59 (16\%) patients: classification of epilepsy in $27(7 \%)$, diagnosis of another condition in $12(3 \%)$, and influenced clinical decision in $20(5 \%)$. The request was justifiable in $104(28 \%)$ and inappropriate in 205 $(56 \%)$. Table 1 shows the comparative figures for the regional centre. These indicate that this test is of greatest value in a specialist epilepsy clinic, where most patients have definite epilepsy, and of least value in district general hospitals, where most requests originate from non-specialist clinics.

\section{Prospective audit}

There was a significant change in the requesting practice after intervention $\left(\chi^{2}=33.85, \mathrm{df}=5, \mathrm{P}<0.0001\right)$ mainly because of a relative decrease in requests by physicians (table 2). Similarly there was a significant change in the nature of requests $\left(\chi^{2}=21.90, \mathrm{df}=6\right.$, $\mathrm{P}=0.0013)$ mainly because of a reduction in the number of requests in patients with funny turns or aggressive outbursts (table 2). There was a concomitant change in the distribution of EEG results $\left(\chi^{2}=11.12\right.$, $\mathrm{df}=3, \mathrm{P}=0.01$ ), where the relative proportion of non-

Table 1 Retrospective audit: utility of EEG by source of request. Figures are number (percentage) of procedures

\begin{tabular}{lccc} 
& $\begin{array}{c}\text { District general hospital } \\
(\mathbf{n = 3 6 8 )}\end{array}$ & $\begin{array}{c}\text { Neurology centre } \\
(\mathbf{n}=\mathbf{7 4})\end{array}$ & $\begin{array}{c}\text { Regional epilepsy clinic } \\
(\mathbf{n = 6 9 )}\end{array}$ \\
\hline Influenced management & $59(16.0)$ & $14(18.9)$ & $23(33.3)$ \\
\hline Justifiable & $104(28.3)$ & $42(56.8)$ & $38(55.1)$ \\
\hline Inappropriate & $205(55.7)$ & $18(24.3)$ & $8(11.6)$
\end{tabular}


Table 2 Change in requesting practice by clinician and by reason for request from retrospective audit (6 months) to prospective audit (6 months). Figures are number (percentage) of procedures

\begin{tabular}{|c|c|c|}
\hline & Retrospective ( $n=368$ ) & Prospective $(\mathrm{n}=241)$ \\
\hline \multicolumn{3}{|l|}{ Requesting clinician: } \\
\hline General practitioner & $17(4.6)$ & $17(7.1)$ \\
\hline Neurologist & $42(11.4)$ & $59(24.5)$ \\
\hline Physician & $168(45.6)$ & $63(26.1)$ \\
\hline Paediatrician & $65(17.7)$ & $55(22.8)$ \\
\hline Psychiatrist & $71(19.3)$ & $42(17.4)$ \\
\hline Other & $5(1.4)$ & $5(2.1)$ \\
\hline \multicolumn{3}{|l|}{ Reason: } \\
\hline Epilepsy & 67 (18.2) & $68(28.2)$ \\
\hline Seizures & $48(13.0)$ & $35(14.5)$ \\
\hline Funny turns & $156(42.4)$ & $83(34.5)$ \\
\hline Aggressive outbursts & $12(3.3)$ & $3(1.2)$ \\
\hline Rapid dementia/ acute confusion* & $8(2.2)$ & $14(5.8)$ \\
\hline Mental symptoms & $42(11.4)$ & $14(5.8)$ \\
\hline Other & $35(9.5)$ & $24(10.0)$ \\
\hline
\end{tabular}

Table 3 Change in distribution of results and usefulness of EEG from retrospective audit (6 months) to prospective audit (6 months). Figures are number (percentage) of procedures

\begin{tabular}{lcc}
\hline \multicolumn{1}{l}{ Diagnosis } & Retrospective $(\mathbf{n}=\mathbf{3 6 8})$ & Prospective $(\mathbf{n}=\mathbf{2 4 1})$ \\
\hline Normal & $190(51.6)$ & \\
\hline Non-specific abnormality & $88(23.9)$ & $36(55.6)$ \\
\hline Epileptiform discharges & $40(10.9)$ & $42(17.5)$ \\
\hline Miscellaneous abnormalities & $50(13.6)$ & $29(12.0)$ \\
\hline Usefulness & $59(16.0)$ & $70(29.0)$ \\
\hline Influenced management & $104(28.3)$ & $86(35.7)$ \\
\hline Justifiable & $205(55.7)$ & $85(35.3)$ \\
\hline Inappropriate & &
\end{tabular}

specifically abnormal results fell considerably (table 3). Table 3 also shows the change in usefulness of EEG $\left(\chi^{2}=26.99, \mathrm{df}=2, \mathrm{P}<0.0001\right)$.

\section{Discussion}

This retrospective audit of use of EEG in a district general hospital showed clear differences in requesting practice between clinicians. A large proportion of these requests were considered to be inappropriate because of the prevalent misconception that the EEG is a useful diagnostic tool in various clinical settings, notably in patients with "funny turns." A secondary finding was that a quarter of these procedures showed non-specific abnormalities. While this study did not specifically examine the issue, evidence exists that overinterpretation of these minor abnormalities contributes to erroneous diagnosis of epilepsy. ${ }^{11} 1415$

The EEG department at the hospital where this study was carried out is staffed by a full time EEG technician, access to the test is unrestricted, and records are reported by a local general practitioner. Before and including the period of the retrospective audit, the number of procedures performed (about 800 a year) was consistent that seen in a survey in the Thames region carried out by the Association of British Clinical Neurophysiologists. ${ }^{4}$ This service, and its use, is typical of that seen in district general hospitals throughout the United Kingdom and the results of this original work are likely to be generalisable.
What is already known on this topic

There is unrestricted access to EEG in most district general hospitals throughout the United Kingdom

The combination of equivocal symptoms and non-specific abnormalities carries a risk of misdiagnosis of epilepsy

\section{What this study adds}

An audit of requests for EEG showed that a large proportion were inappropriate, mainly because of the prevalent misconception that the procedure could prove or exclude a diagnosis of epilepsy in patients with "funny turns"

After intervention with clinicians, which used an educative approach, there was a considerable and sustained change in requesting practice

\section{Intervention}

The intervention involved an educative, nonconfrontational approach with time taken to demonstrate to clinicians the limitations and pitfalls of the interictal EEG and that in patients with "funny turns" they had usually achieved a diagnosis on clinical grounds alone without the aid of EEG. That the prospective audit was successful reflects the quality of communication and cooperation between investigating team and the users of the service.

After the intervention there were fewer requests for EEG from general physicians, particularly for patients with funny turns. This should result in less chance of clinicians being in the difficult position of decision making in patients with "equivocal symptoms and non-specific EEG abnormalities" which, consequently, may reduce misdiagnosis of epilepsy. Furthermore, concomitant with the reduction in unnecessary EEG requests, there was an increase in the proportion of procedures that directly influenced management of patients.

We attribute the observed changes in practice to the intervention. Firstly, the proportion of inappropriate $(35 \%)$ and justifiable $(36 \%)$ requests in the prospective audit make it unlikely that there were many instances of EEG not being requested when it should have been. Secondly, the requesting clinicians continued to refer to the general hospital department only. Thirdly, if the change occurred without any understanding of the project, the effect would not be sustained. However a "user friendly" standardised EEG form, incorporating the "indications" element of the guidelines was introduced in October 1996 and the number of requests has continued to fall. In the three years before the audit there were 2485 requests (936 in 1992, 756 in 1993, and 793 in 1994), whereas in the three years after the audit there were 1351 requests (510 in 1997, 475 in 1998, and 366 in 1999).

\section{Conclusions}

We have shown that by using an educative approach sustainable change in practice can be achieved, which has benefits for patients, clinicians, and service provision. Through greater understanding of its limita- 
tions clinicians have adopted a more selective EEG requesting policy. Doctors may have a lower risk of making, and patients may have lower risk of receiving, a misdiagnosis of epilepsy. The reduction in the number of unnecessary procedures releases technical capacity which can be used in the conduct of other investigations. Systematic replication of this work, possibly on a regional basis, would yield savings which would permit development of accessible local neurophysiology services.

Julie Jones and Clare Jowett in the audit departments of Wrexham Maelor and Royal Shrewsbury Hospitals facilitated the meetings and distribution of guidelines. We specially thank the users of the service for listening and changing their practice

Contributors: DS had the original idea for the audit, helped with data collection and analysis, and is guarantor for the study. $\mathrm{RB}$ did most of the data collection. RP reported on all the EEGs.

The paper was jointly written by DS, RB, RP, and BT.

Funding: None.

Competing interests: DS presented the data at an educational meeting sponsored by GlaxoWellcome, for which he received payment

1 Fowle AJ, Binnie CD. Uses and abuses of the EEG in epilepsy. Epilepsia 2000:41(suppl 3):10-8.

2 Commission on classification and terminology of an international league against epilepsy. Proposal for classification of epilepsies and epileptic syndromes. Epilepsia 1989;30:389-99.
3 King MA, Newton MR, Jackson GD, Fitt GJ, Mitchell LA, Silvapulle MJ, et al. Epileptology of the first seizure presentation; a clinical, electroencephalographic and magnetic resonance imaging study of 300 consecutive patients. Lancet 1998;352:1007-11.

4 Binnie CD, Prior PF. Electroencephalography. J Neurol Neurosurg Psychia try 1994;57:1308-19.

5 Berg AT, Shinnar S. The risk of recurrence following a first unprovoked seizure. Neurology 1991;41:965-72.

6 MRC Antiepileptic Drug Withdrawal Study Group. Prognostic index for recurrence of seizures after remission of epilepsy. BMJ 1993;306:1374-8.

7 Binnie CD. Electroencephalography. In: Laidlaw J, Richens A, Chadwick D, eds. A textbook of epilepsy. 4th ed. Edinburgh: Churchill Livingstone,1992:277-8.

8 Ajmone-Marsan C, Zivin LS. Factors related to the occurrence of typical paroxysmal abnormalities in the EEG records of epileptic patients. Epilepsia 1970;11:361-81.

9 Bridgers SL. Epileptiform abnormalities discovered on electroencephalographic screening of psychiatric in-patients. Arch Neurol 1987;44:312-6.

10 Gregory RP, Oates T, Merry RTG. Electroencephalogram epileptiform abnormalities in candidates for aircrew training. Electroencephalogr Clin Neurophysiol 1993;86:75-7

11 Riley TL. Normal variants in EEG that are mistaken as epileptic patterns. In: Gross M, ed. Pseudoepilepsy. Lexington KY: Heath, 1983:25-7.

12 Nicolaides P, Appleton RE, Beirne EM. EEG requests in paediatrics: an audit. Arch Dis Child 1995;72:522-3.

13 Binnie CD. EEG audit: increasing cost efficiency of investigations in epilepsy. Electroencephalogr Clin Neurophysiol 1990;76:29P.

14 Gibbs J, Appleton RE. False diagnosis of epilepsy in children. Seizure 1992;1:15-8.

15 Smith D, Dafalla B, Chadwick DW. The misdiagnosis of epilepsy and the management of refractory epilepsy in a specialist clinic. QJ Med 1999; $15-23$.

(Accepted 14 February 2001)
In contrast to North America and Australia, little attention has been paid to the use of health services by adolescents in the United Kingdom. The incidence of survival from chronic illness in young people is increasing. The care of adolescents is becoming a quality issue for the NHS. ${ }^{12}$ Health data are rarely available in the United Kingdom on adolescents as a separate group, with standard data dividing young people into those aged under 14 years or those aged 15-44 years. ${ }^{3}$ A study of the use of psychiatric beds by adolescents in England and Wales is presently being undertaken by the Royal College of Psychiatrists (A O'Herlihy, personal communication). Previous regional studies have been undertaken, ${ }^{45}$ but reliable national data to guide the provision of other hospital services to adolescents are not available.

\section{Participants, methods, and results}

I requested information on the numbers of hospital bed days of inpatients and day case patients aged 12 to 19 years from April 1997 to March 1998 from all health authorities and boards in Wales and Scotland and 27 randomly selected ones from England. I excluded admissions for obstetrics, mental health, and learning disabilities. Information was provided by 37 health authorities and boards (response rate 79\%), including three of the five in Wales, all 15 health boards in Scotland, and 19 English health authorities and boards, including at least one from each of the eight English regions. Information from three health authorities and boards was unusable. Average bed days was calculated by summing data from all 34 included health authorities and boards (population 15.8

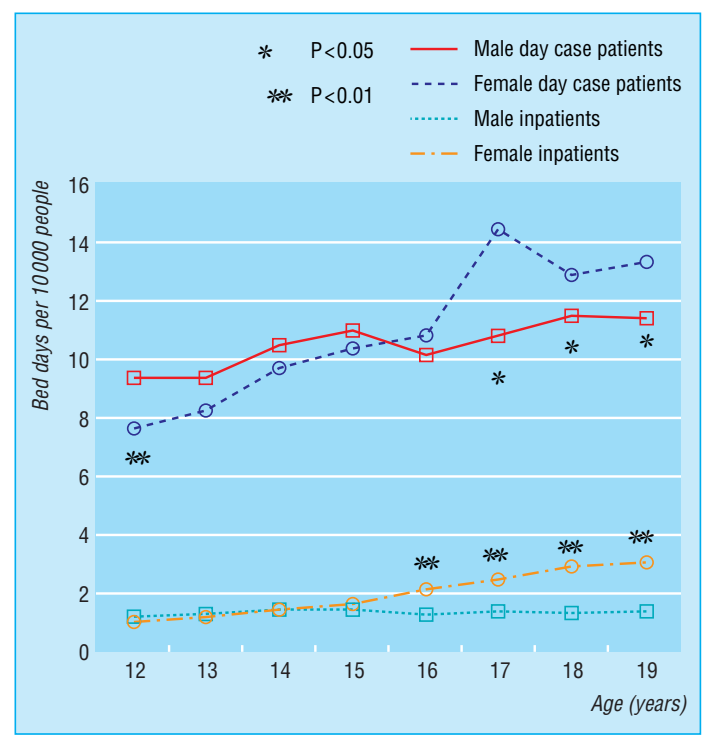

Bed days of adolescent inpatients and day case patients per 10000 population by age and sex
Editorial by Macfarlane and Blum

Department of Medicine, University College London, London W1T 3AA

R M Viner adolescent medicine and endocrinology R.Viner@ich. ucl.ac.uk

BMJ 2001;322:957-8 